\title{
Stephen Y. Chan: perseverance through criticism is essential for success in cardiovascular research
}

Submitted Jul 30, 2018. Accepted for publication Jul 31, 2018.

doi: $10.21037 /$ cdt.2018.08.02

View this article at: http://dx.doi.org/10.21037/cdt.2018.08.02

\section{Editor's note}

Rarely is a disease induced by an anomaly in one single gene, but an issue of various pathobiological processes interacting in a complex intra and intercellular network. Network medicine, a term originated from a scientific article "Network Medicine-From Obesity to the "Diseasome" authored by Barabási (1), has given scientific research a new perspective by offering a platform to not only burrow into the molecular complexity of a particular disease, but also the molecular relationships among apparently distinct phenotypes (2). It is believed that, specifically in the study of pulmonary hypertension, traditional methods of discovery are neither able to address the root causes of the disease nor its variations across different people.

Over the years, Prof. Stephen Y. Chan, Associate Professor of Medicine, Division of Cardiology, University of Pittsburgh Medical Center, Pittsburgh, Pennsylvania, United States (Figure 1), has been delving into the molecular mechanisms of pulmonary vascular disease and pulmonary hypertension. Through capitalizing on the emerging discipline "network medicine", his team is putting sustained effort in the definition of the network biology of non-coding RNAs in pulmonary hypertension, the study of the molecular regulation of mitochondrial metabolism by microRNAs, and the definition of the regulation of circulating microRNAs in hypoxia and exercise. Cardiovascular Diagnosis and Therapy is pleased to invite Prof. Chan for an exclusive interview in which he will share with us useful advice and highlight some of his most interesting findings in his career thus far in cardiovascular research.

\section{Expert's introduction}

Stephen Y. Chan, MD, PhD, FAHA, is the Associate Professor of Medicine, Division of Cardiology at the University of Pittsburgh Medical Center, Pittsburgh,

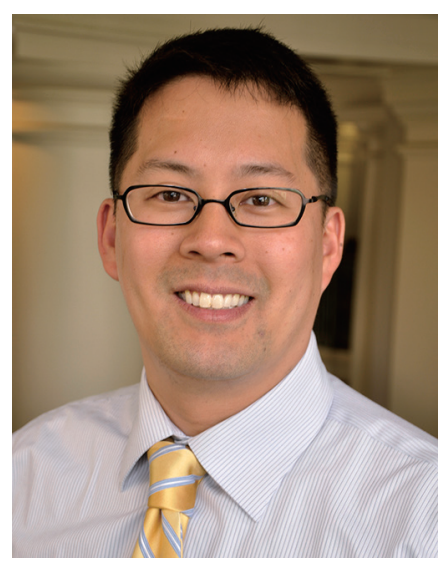

Figure 1 Prof. Stephen Y. Chan.

Pennsylvania, United States. There he also directs the Center for Pulmonary Vascular Biology and Medicine, and serves as the Associate Fellowship Director in Cardiology. Graduating from the Massachusetts Institute of Technology and receiving his $\mathrm{MD}$ and $\mathrm{PhD}$ from the University of California, San Francisco, Prof. Chan further completed an internship and residency in Internal Medicine at the Brigham and Women's Hospital (BWH) and fellowship training in Cardiology at the Massachusetts General Hospital.

Prof. Chan has been actively involved in basic and translational research with a focus on the molecular mechanisms of pulmonary vascular disease and pulmonary hypertension. His research projects have been receiving funding support by the National Institutes of Health, the American Heart Association, the Pulmonary Hypertension Association, and Gilead Sciences. He has received a number of philanthropic awards at BWH, including the Lerner Scholarship, the Watkins Discovery Award, the Harris Family Research Prize, and the McArthur-Radovsky Research Scholarship, as well as several international research awards from the American College of Cardiology, the American Heart Association, and the American Society of Microbiology. 


\section{Interview questions}

CDT: Your research has been capitalizing on "network medicine" to accelerate discovery in pulmonary hypertension. What is the significance of the use of network theory to discover $R N A$-based origins of human diseases?

Prof. Chan: My laboratory is driven by the concept that traditional discovery methods in pulmonary hypertension $(\mathrm{PH})$ are hampered by an inability to study the initial stages of disease and have failed to produce treatments that address the root causes of disease. Current medications for $\mathrm{PH}$ do not prevent or reverse disease. They are based on a onesize-fits-all model, and address neither the root causes nor the variations of $\mathrm{PH}$ across different people. We know little about the nature of the initiating injuries in $\mathrm{PH}$. This stems from two facts: an inability to recognize the early stages of disease and an inability to remove lung blood vessels from a living person until late in disease when death or lung transplantation allows for tissue harvest. By that time, so many molecular processes are altered, it is nearly impossible to deduce the initiating injuries-injuries that may have occurred years or decades before the final disease state was reached. Yet, if such insight could be achieved, we could develop treatments specific to the type of $\mathrm{PH}$, thus making disease prevention or a cure a reality.

We believe that the inability to study directly the initial stages of PH may be overcome through mathematical methods to analyze big data. In my laboratory, we utilize "network theory" to construct computational models of $\mathrm{PH}$ to perform high-volume, systematic analyses that are not feasible with standard experimentation. These analyses then guide our laboratory-based experiments in mice and humans as definitive validation of our work. This allows us to consider entire molecular profiles from specific $\mathrm{PH}$ patients without making incorrect generalizations about the population as a whole. My laboratory has already had success using this approach in establishing the crucial importance of a set of molecules called microRNAs (i.e., small pieces of genetic material) in $\mathrm{PH}$ progression. With further application, we believe that this approach will facilitate predictions regarding the elusive origins of $\mathrm{PH}$ that have not been possible to this point.

CDT: In your study, you bave identified a few hypoxamirs in pulmonary vascular function. What roles do they actually play in the pathogenesis of $\mathrm{PH}$ ?

Prof. Chan: We have employed the study of microRNAs
(miRNAs) —non-coding RNAs that regulate multiple gene transcripts simultaneously in a given cell—as a platform for such network-based discovery. Our work was the first to identify the crucial systems-level functions of microRNAs in PH. For example, we identified the hypoxia-induced miR-210 as an essential regulator of oxidative metabolic reprogramming in both the conserved hypoxic response in all mammalian cells and in PH. Louis Pasteur first described a metabolic shift from mitochondrial oxidative phosphorylation to glycolysis as a fundamental cellular response to hypoxia. This "Pasteur effect" profoundly influences cell survival and function, but its underlying molecular mechanisms have been incompletely understood. At the time of our study, our understanding of microRNA biology was still developing, and microRNAs had not yet been implicated in the control of mitochondrial metabolism at any level. We identified the hypoxia-induced microRNA-210 as an essential regulator of the metabolic processes that govern the Pasteur effect, via repression of its direct target, the iron-sulfur cluster assembly proteins ISCU1/2, repressing iron-sulfur (Fe-S) biogenesis, and perturbing mitochondrial electron transport and respiration during the acute response to cellular hypoxia (3). In vivo, we also demonstrated that, driven by acquired (hypoxia) or genetic causes in animals and humans, the miR-210-ISCU1/2 regulatory axis is a pathogenic lynchpin causing Fe-S deficiency and PH. In doing so, we defined a new human cohort of patients carrying genetic mutations of ISCU1/2 who suffer from exercise-induced $\mathrm{PH}$ (4). These findings carry broad translational implications. Identifying patients early with mutations in other Fe-S biogenesis genes may offer the ability to prevent $\mathrm{PH}$. Design of new PH drugs can now focus on miR-210, ISCU1/2, and/ or other genes known to be linked to Fe-S biology but have yet to be studied in PH. Finally, there likely exist medical conditions beyond $\mathrm{PH}$ ranging from heart disease, cancer, and neurologic disease that may share a dependence on Fe-S generation and could be treated similarly.

\section{CDT: What are your latest findings in the study of the molecular mechanisms of pulmonary vascular disease and $\mathrm{PH}$ ?}

Prof. Chan: Building upon the work with hypoxia-relevant miRNAs, we have also been the first to demonstrate the validity of network theory in predicting in vivo pulmonary vascular function. We developed an in silico approach to rank microRNAs by their targeting patterns of known networks of $\mathrm{PH}-$ relevant genes. By coupling these efforts to experimental validation in both animal and human $\mathrm{PH}$ models, we identified 
the top-ranked microRNA family, miR-130/301, as a master regulator of multiple subordinate microRNA and target gene pathways with coordinated effects on proliferation, vasomotor tone, and vascular stiffness in $\mathrm{PH}(5,6)$.

More recently, stemming from our insights in miRNA biology, we found that vascular stiffness and extracellular matrix (ECM) remodeling are not merely end-stage disease consequences but rather occur early in $\mathrm{PH}$ and promote pulmonary vascular crosstalk and dysfunction. We found that mechanoactivation of the YAP/TAZ transcriptional coactivation factors along with the miR-130/301 family controls a feedback loop to propagate matrix stiffening throughout the pulmonary vascular tree which in turn controls glutamine metabolism to sustain proliferating vascular cells. These findings paved the way for my lab to re-purpose drugs for $\mathrm{PH}$ that inhibit YAP or glutamine metabolism and for us to explore vascular stiffness as a direct mediator of inflammation, metabolism, and pulmonary vascular cell identity (7).

\section{CDT: What are the technical challenges faced in your study?}

Prof. Chan: There exist multiple technical challenges in the study of pulmonary hypertension. Namely, there remains an inability to remove lung blood vessels from a living person until late in disease when death or lung transplantation allows for tissue harvest. As such, we have very little direct access to study the early stages of disease. Animal models of $\mathrm{PH}$ recapitulate some but not all features of human disease. To make substantial discovery in this field, therefore, we are compelled to utilize multiple independent lines of experimental approach to reach our conclusions-merging computational discovery and omics data, with cell culture, animal modeling, and human translational work. It has taken nearly a decade to build that type of infrastructure in our program, but we finally feel that our program has this type of capability to make transformative discoveries in the field over the next 5-10 years.

CDT: Your studies have been holding research grants from NIH and many other funding institutions, would you introduce us to a recent research project that you are involved in?

Prof. Chan: We recently were funded by the NIH to work on a project regarding the mechanisms by which human immunodeficiency virus (HIV) can cause pulmonary arterial hypertension (PAH). Increasing evidence suggests that $\mathrm{PAH}$ is more common in HIV cohorts than originally anticipated Yet, the molecular origins of HIV-PAH remain entirely enigmatic, and treatments are limited. Based on our recently reported new paradigms in $\mathrm{PAH}$-namely, that vascular extracellular matrix (ECM) remodeling, vessel stiffening, and vascular glutamine metabolism are early, linked processes that promote various types of PAH, we hypothesized that these processes govern a cell-cell communication between infected immune cells and the pulmonary vascular cells to lead to vascular stiffening, altered glutamine metabolism, and ultimately HIV$\mathrm{PAH}$. Thus, our project aims were to determine how this putative cell-cell communication operates, whether such pathogenic axes are evident in humans with HIV-PAH, and whether pharmacologic interruption of glutamine metabolism may improve $\mathrm{PAH}$ in a primate model of simian immunodeficiency virus (SIV)-induced PAH. We are excited about this project as we are leveraging the only known reliable animal model of HIV-PAH in conjunction with direct human studies, thus ensuring both mechanistic insight and applicability to this form of human PAH to an extent that has never been possible before. Perhaps most importantly, it offers a rare opportunity to establish a much needed targeted therapeutic for this historically neglected vascular disease.

\section{CDT: Having studied pulmonary vascular disease and pulmonary bypertension for years, how did you become involved in this line of research?}

Prof. Chan: I first became interested in pulmonary hypertension and pulmonary vascular disease as a clinical fellow in cardiology caring for these patients. In those days, we had little medical therapy to offer these patients, and this diagnosis was uniformly fatal without heart/lung transplantation. So, there was a great need for advances in the field. Coupled with that, I was intrigued by the clinical observations that so many seemingly disparate medical conditions all are associated and converge as risk factors for pulmonary vascular disease-HIV infection, autoimmune disease, congenital heart disease, sleep apnea, pulmonary embolism, BMPR2 mutations, among many others. It was, and still is, a fascinating puzzle that has major implications for the health and well-being of our patients and their families. I felt that this was a field of study and career that was well worth my investment.

CDT: What do you regard as the most interesting aspects of research? What have been driving you to move forward 


\section{in your research field?}

Prof. Chan: Being a physician-scientist (I am both a practicing cardiologist and basic and translational researcher) has been and continues to be a wonderful career choice for me. The aspect that I appreciate the most is the variety that comes with my daily and weekly routine. I have the opportunity to work directly with patients, to teach and mentor clinical and research trainees alike, to design and innovate into problems that never before have had an answer, and to run major clinical and research programs. Coupled with the fact that I truly believe that our work will have positive impact on the lives of our patients, I could not imagine being happier with my career choice. Certainly, this career path came with sacrifice in terms of financial compensation, long years of training and work, and a path that is not a "sure bet" (research never is). But, anything that is worth doing requires risk and sacrifice-I'm very happy that I had the opportunity for both.

\section{CDT: As an experienced researcher, what do you think is the key to successful research?}

Prof. Chan: In cardiology, this is an important question, as we continue to see physician-scientist numbers dwindle. I have found that many of our trainees cite the fact that there is so much sacrifice and uncertainty in a career of research. While I agree with that, I also think there are strategies that dramatically increase your chances for success. First is perseverance. Rejection and criticism are a staple in research investigation - the most successful scientists are the ones that can discern the constructive nature of criticisms (even when they are harsh) and make corrections but do not otherwise take those experiences too personally. Second is choosing a mentor(s) wisely. You cannot and should not try to go alone in this path-having mentors with experience and who care about you is critical to success at every stage. Third is do not be afraid to re-invent yourself as a scientist, to learn new things, and to go in completely new directions when the biology and directions take you there. Fourth, learning how to multi-task well can be a survival skill and can serve you well. Fifth is surround yourself with a team that is smarter and more capable than you. This is particularly true when you run your own lab/group and you are managing rather than doing the experiments yourself.

\section{Acknowledgements}

We would like to express our sincerest gratitude to Prof. Stephen Y. Chan for sharing his insights and opinions with us.

\section{Footnote}

Conflicts of Interest: The author has no conflicts of interest to declare.

\section{References}

1. Barabási AL. Network Medicine - From Obesity to the "Diseasome". N Engl J Med 2007;357:404-7.

2. Barabási AL, Gulbahce N, Loscalzo J. Network medicine: a network-based approach to human disease. Nat Rev Genet 2011;12:56-68.

3. Chan SY, Zhang YY, Hemann C, et al. MicroRNA-210 controls mitochondrial metabolism during hypoxia by repressing the iron-sulfur cluster assembly proteins ISCU1/2. Cell Metab 2009;10:273-84.

4. White K, Lu Y, Annis S, et al. Genetic and hypoxic alterations of the microRNA-210-ISCU1/2 axis promote iron-sulfur deficiency and pulmonary hypertension. EMBO Mol Med 2015;7:695-713.

5. Bertero T, Cotrill KA, Lu Y, et al. Matrix remodeling promotes pulmonary hypertension through feedback mechanoactivation of the YAP/TAZ-miR-130/301 circuit Cell Rep 2015;13:1016-32.

6 Bertero T, Lu Y, Annis S, et al. Systems-level regulation of microRNA networks by miR-130/301 promotes pulmonary hypertension. J Clin Invest 2014;124:3514-28.

7. Bertero T, Oldham WM, Cottrill KA, et al. Vascular stiffness mechanoactivates YAP/TAZ-dependent glutaminolysis to drive pulmonary hypertension. J Clin Invest 2016;126:3313-35.

(Science Editor: Brad Li, CDT, editor@thecdt.org)
Cite this article as: $\mathrm{Li}$ B. Stephen Y. Chan: perseverance through criticism is essential for success in cardiovascular research. Cardiovasc Diagn Ther 2018;8(4):559-562. doi: 10.21037/cdt.2018.08.02 